\title{
Assessing the effects of a sequestered germline on interdomain lateral gene transfer in Metazoa
}

\author{
Lindy Jensen, ${ }^{1,2}$ Jessica R. Grant, ${ }^{1}$ Haywood Dail Laughinghouse IV, ${ }^{1}$ and Laura A. Katz ${ }^{1,3,4}$ \\ ${ }^{1}$ Department of Biological Sciences, Smith College, Northampton, Massachusetts 01063 \\ ${ }^{2}$ Current Address: Department of Molecular and Integrative Physiology, University of Michigan, Ann Arbor, Michigan
} 48109

${ }^{3}$ Program in Organismic and Evolutionary Biology, University of Massachusetts, Amherst, Massachusetts 01003

${ }^{4}$ E-mail: Ikatz@smith.edu

Received March 20, 2015

Accepted April 19, 2016

\begin{abstract}
A sequestered germline in Metazoa has been argued to be an obstacle to lateral gene transfer (LGT), though few studies have specifically assessed this claim. Here, we test the hypothesis that the origin of a sequestered germline reduced LGT events in Bilateria (i.e., triploblast lineages) as compared to early-diverging Metazoa (i.e., Ctenophora, Cnidaria, Porifera, and Placozoa). We analyze single-gene phylogenies generated with over 900 species sampled from among Bacteria, Archaea, and Eukaryota to identify well-supported interdomain LGTs. We focus on ancient interdomain LGT (i.e., those between prokaryotes and multiple lineages of Metazoa) as systematic errors in single-gene tree reconstruction create uncertainties for interpreting eukaryote-to-eukaryote transfer. The breadth of the sampled Metazoa enables us to estimate the timing of LGTs, and to examine the pattern before versus after the evolution of a sequestered germline. We identified 58 LGTs found only in Metazoa and prokaryotes (i.e., bacteria and/or archaea), and seven genes transferred from prokaryotes into Metazoa plus one other eukaryotic clade. Our analyses indicate that more interdomain transfers occurred before the development of a sequestered germline, consistent with the hypothesis that this feature is an obstacle to LGT.
\end{abstract}

KEY WORDS: Animals, gene loss, horizontal gene transfer, lateral gene transfer, Metazoa, weak link model.

\begin{abstract}
Lateral gene transfer (LGT), the non-vertical exchange of genetic material from one lineage to another, has had a substantial impact on biological diversity on Earth (Davison 1999; Gogarten et al. 2002; Jain et al. 2003; Dagan and Martin 2009; Katz 2012; Syvanen 2012; Soucy et al. 2015; Katz 2015). Although LGTs within and between bacteria and archaea are relatively common (Doolittle 1999; Nelson et al. 1999; Ochman et al. 2000; Grant and Katz 2014), the contributions of interdomain LGT from bacteria or archaea into eukaryotes is less well understood (Andersson 2005; Keeling and Palmer 2008; Syvanen 2012; Wijayawardena et al. 2013; Boto 2014). In particular, the contribution of interdomain LGT across the metazoan tree of life is unclear due to a dearth of taxon-rich analyses that include both metazoan and bacterial/archaeal lineages.
\end{abstract}

There are numerous approaches for distinguishing laterally transferred genes from vertically inherited genes (i.e., genes passed down within a lineage). One approach is to analyze phylogenetic trees generated from homologs sampled from diverse taxa, and then examine the results for incongruence (Keeling and Palmer 2008). A gene tree is identified as incongruent when the evolutionary relationships between the species are discordant with established phylogenetic relationships, and LGT provides a parsimonious explanation for the incongruence (Keeling and Palmer 2008; Dagan 2011). Although this methodology is appropriate for identifying ancient LGT events, limited or biased taxon sampling and poor data quality can be problematic in interpreting patterns in single-gene trees (Ragan et al. 2006). Fortunately, the growing availability of whole genomes is alleviating these shortcomings. 
An alternative to gene tree analyses is to use methods that assess patterns in sequence composition, though these methods work best for recent events. For example, differences in the composition of one gene compared to another or codon frequencies can be compared (Ragan 2001; Lawrence and Hendrickson 2003). Although these methods often yield larger numbers of anomalies and do not require as broad a taxon sampling, these parametric methods have their own set of disadvantages as distinguishing causes for changes in compositional bias can be challenging. Most importantly for the current study, foreign genes begin to resemble the genome around them over time and gene loss/duplication can confound results (Lawrence and Ochman 2002). As a result, studies examining the same data but using different parameters to generate different sets of putative LGTs can yield conflicting results (Ragan et al. 2006).

After finding laterally transferred genes, determining the function of these genes can reveal the role of LGT within lineages. Laterally transferred genes arguably confer adaptive functions and novel traits (Schönknecht et al. 2013), the most notable example being the massive endosymbiotic gene transfers (EGTs) in eukaryotes from mitochondria and plastids (Katz 2002; Andersson 2005; Keeling and Palmer 2008). However, outside of EGT, laterally transferred genes found in eukaryotes tend to be metabolic genes (Jain et al. 1999, 2003; Koning et al. 2000; Keeling and Palmer 2008). Similarly, in bacteria many LGTs are involved in metabolic functions (Pál et al. 2005; Whitaker et al. 2009; Lehmann and Richardson 2010). Dubbed "the complexity hypothesis," Jain et al. (1999) proposed that the "operational" genes often involved in cellular metabolism are much more likely to be successfully transferred than "informational" genes that are part of complex networks. In other words, metabolic and housekeeping processes that only involve two or three protein interactions are more likely to be successfully exchanged among lineages (Whitaker et al. 2009).

The impact of LGT in Metazoa has been assumed to be low based on the hypothesis that the sequestered metazoan germline is an obstacle to LGT (Doolittle 1999; Andersson et al. 2001; Katz 2002; Andersson 2005; Keeling and Palmer 2008; Boto 2010; Huang 2013; Schönknecht et al. 2014). However, not all Metazoa have a sequestered germline, as germline determination is more fluid among early-diverging lineages (i.e., Ctenophora, Cnidaria, Porifera, and Placozoa). The absence of a sequestered germline in early-diverging Metazoa might lead to an increase in LGT rates because: (1) germ cells are more accessible to foreign DNA throughout their lifespan; and (2) the presence of totipotent cell lines that can take on a variety of fates during the life cycle (including gametes) provides more opportunities for transfer (Funayama 2010; Juliano et al. 2010; Alie et al. 2011; David 2012). Germ cells of some early-diverging metazoan lineages often move throughout the body or line the inside of the gut (Simpson 1980, 1984; Gaino et al. 1984, 1986; Brusca and Brusca 2003; Nishimiya-Fujisawa and Kobayashi 2012). In contrast, germ cells in Bilateria (i.e., triploblasts) generally migrate during development to a specific location away from the gut where they are sheltered from foreign DNA by layers of tissue (Starz-Gaiano and Lehmann 2001; Extavour 2007; Ewen-Campen et al. 2010; Lehmann and Richardson 2010). Among Bilateria, it is generally only the primordial germ cells (PGCs) that are capable of differentiating into gametes, and somatic cells have no gametogenic potential (Extavour and Akam 2003).

Numerous studies have explored laterally transferred genes in metazoan lineages demonstrating that LGT is at least possible in lineages with and without sequestered germline (Kondrashov et al. 2006; Gladyshev et al. 2008; Starcevic et al. 2008; Klasson et al. 2009; Nikoh and Nakabachi 2009; Danchin et al. 2010; Paganini et al. 2012; Sloan and Moran 2012; Boschetti et al. 2012; Cobbs et al. 2013; Crisp et al. 2015). Perhaps the most wellknown example stems from the symbiotic relationship involving arthropods and nematodes and the bacterial genus Wolbachia, which has been shown to be a donor of laterally transferred genes in diverse insect lineages (Dunning Hotopp 2011). In nematodes, LGT may have played a key role in the development of plant parasitism (Haegeman et al. 2011). Laterally transferred genes have also been found in chordates as the potential source for a cellulose synthase gene in Ciona intestinalis (Nakashima et al. 2004). Among early-diverging Metazoa, the biomineralization gene in sponges may be the product of LGT (Jackson et al. 2011). Lastly, 71 genes found in the detailed examination of the Hydra genome had strongly supported LGT origins (Chapman et al. 2010). These studies primarily focus on detecting very recent LGT in a small number of metazoan species.

Here, we assess a set of single gene trees generated by a taxon-rich phylogenomic pipeline (Grant and Katz 2014) to test the hypothesis that the evolution of a sequestered germline reduced the number of LGTs into Bilateria. To diminish the chances of misinterpreting the systematic errors that confound the topologies of individual gene trees at the time scales relevant for this study (i.e., 100's of millions of years), we chose to focus on interdomain "presence/absence" genes. These genes are defined based on their presence only in a monophyletic clade of Metazoa plus prokaryotes (bacteria and/or archaea), and their absence in all other eukaryotes (Fig. 1). The resulting "presence/absence" genes were either (1) present in the last common ancestor of Metazoa and bacteria or archaea, then lost in every other lineage since, or (2) the product of a LGT event. Placing the resulting LGTs onto a metazoan phylogeny allows us to determine the approximate time of lateral transfer for each individual gene. As the evolution of a sequestered germline happened near the time protostomes and deuterostomes diverged (i.e., just at the base of Bilateria), we compared the number of laterally transferred genes in Bilateria 


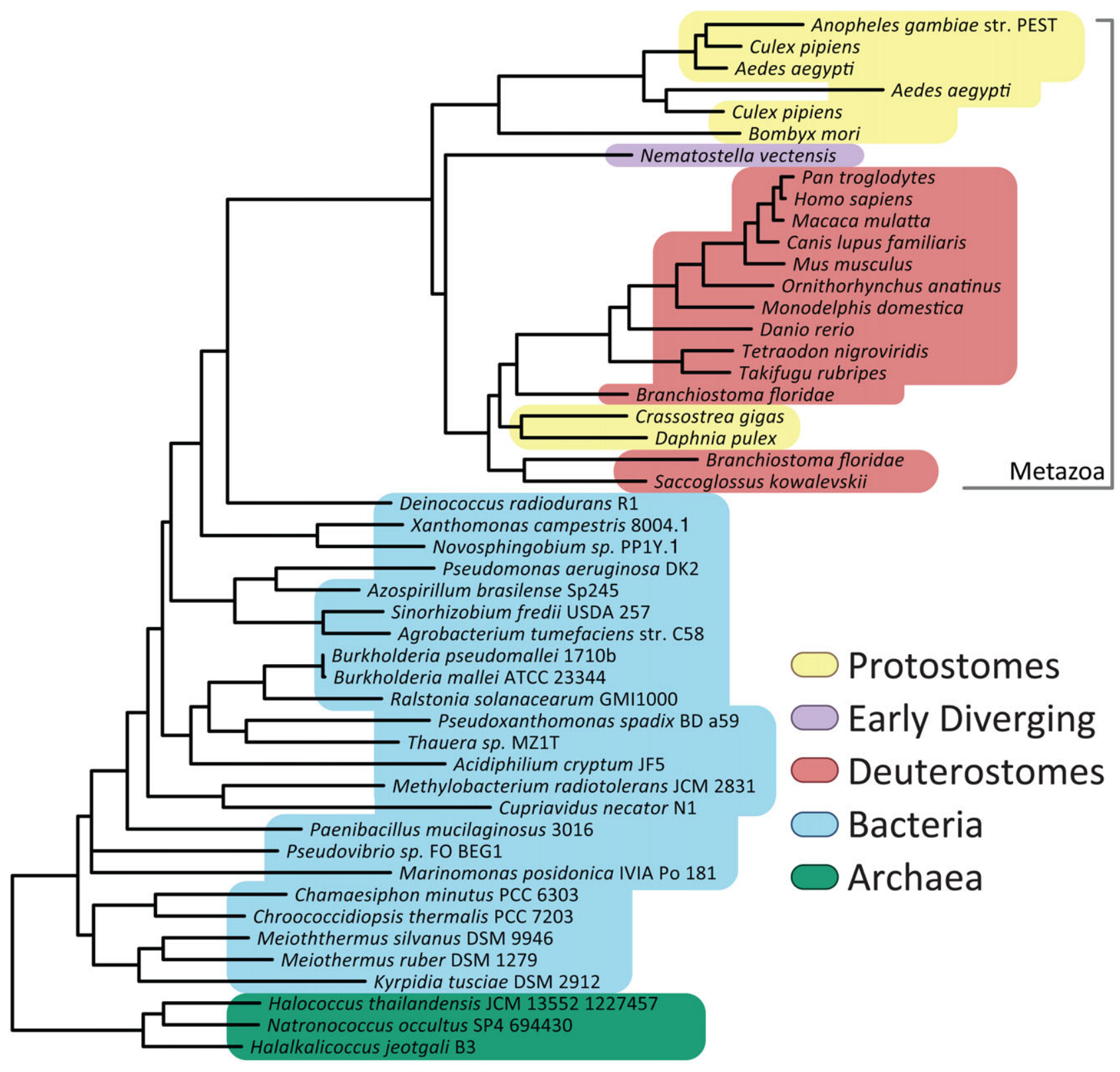

0.2

Figure 1. Example of a gene present only in Metazoa and bacteria/archaea. This single-gene tree is for the gene URAD (Putative 2-oxo4-hydroxy-4-carboxy-5-ureidoimidazoline decarboxylase), a gene involved in a uric acid degradation pathway, which was transferred during event $D$ (see Tables 1 and S2, and Fig. 3). The phylogeny was built from the URAD gene sequences that were present in any of the 910 taxa in our pipeline, serving as an example of a gene present only in Metazoa and bacteria/archaea (e.g., presence/absence data). Colors corresponding to major taxonomic groups are labeled in the bottom right corner.

to the number of genes laterally transferred in early-diverging lineages that lack a sequestered germline.

\section{Methods}

\section{PHYLOGENOMIC PIPELINE}

Single-gene alignments and trees were built using a phylogenetic pipeline (Grant and Katz 2014) with a modified taxonomic dataset that aimed to capture a greater number of animal lineages. In brief, this pipeline starts with set of protein coding genes from taxa of interest, and then uses BLAST (Altschul et al. 1990) to compile them into homologous clusters as determined by OrthoMCL DB (Chen et al. 2006). Then, custom python scripts refined data from each taxon by removing nearly identical sequences (i.e., alleles) and highly divergent sequences (i.e., those sharing only motifs) as described in Grant and Katz (2014). Multisequence alignments 
are generated using Guidance (Penn et al. 2010), which also allows for the automated removal of poorly fitting taxa and columns.

The pipeline includes data from 910 taxa chosen to broadly represent the three domains of life, including 489 eukaryotes, 303 bacteria, and 118 archaea. Metazoa were chosen to capture a broad diversity of lineages, which is necessary to accurately place ancient transfer events in metazoan evolution. At the same time, we sought some level of evenness between the early diverging lineages and the more thoroughly sampled bilaterian lineages to prevent bias from oversampling recent events in one portion of the tree compared to the other. In total, 61 Metazoa were included in this study, of which 25 were early diverging and 36 were bilaterian (19 protostomes and 17 deuterostomes; Table S1). The level of available data varied among lineages with some represented by whole genomes and others by expressed sequence tags (ESTs) or RNAseq data (Table S1).

\section{FINDING PRESENCE/ABSENCE GENES IN ONLY METAZOA AND BACTERIA/ARCHAEA}

We identified a total of 58 genes that are found only in Metazoa and bacteria and/or archaea (i.e., presence/absence data; Fig. 1) from the 1575 alignments that included more than two metazoan taxa and more than two bacterial and/or archaeal lineages (see Fig. $\mathrm{S} 1$ for a schematic of the total process). With the original 1575 trees as a starting point, we used custom Python scripts based on the tree-walking methods in p4 (Price et al. 2009, 2010) to assess the relationships in the tree topologies and identify 310 candidate laterally transferred genes (i.e., genes with Metazoa sister to bacteria and/or archaea). To assess presence/absence data further, we searched that pool of 310 candidates for genes found only in Metazoa and bacteria and/or archaea using additional custom Python scripts (Exemplar tree in Fig. 1). To control for contamination and recent LGT, each gene tree could contain up to two nonsister single eukaryotes (i.e. contaminants), resulting in 193 potential presence/absence genes. The pipeline, which was developed to assess deep phylogenetic relationships, uses a relaxed criterion for sequence and taxon scoring in Guidance (Penn et al. 2010). To increase our confidence in the homology of the presence/absence genes, we realigned the 193 gene sequences with the more stringent default settings for removing non-homologous sequences. This generated a set of 72 putative presence/absence genes. We then eliminated any alignments with fewer than 10 total sequences or only one metazoan species (regardless of the number of paralogs). The remaining 65 alignments were then inspected by eye and an additional six alignments were removed because the alignment quality did not meet our strict standards, for example by having nonmonophyletic Metazoa. This resulted in a final count of 58 presence/absence genes.

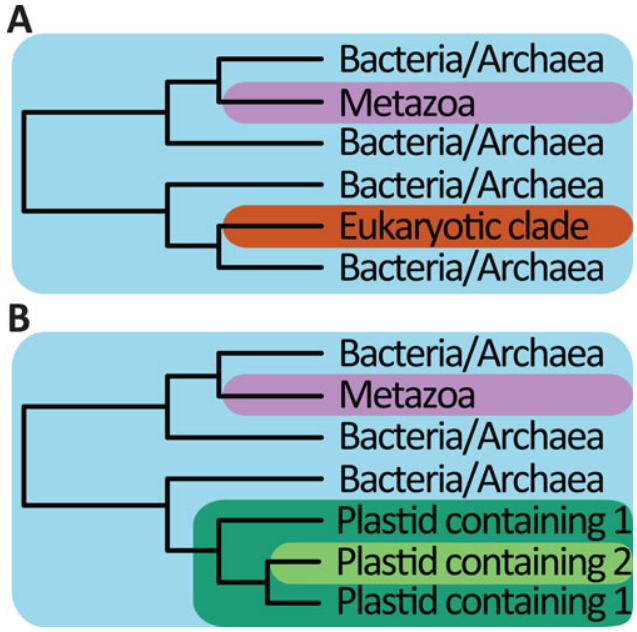

Figure 2. Tree topology trends in genes laterally transferred twice. Diagrams summarizing the two trends found in the topology of genes laterally transferred twice. (A) Describes genes that were also transferred into one other eukaryotic supergroup in addition to a transfer into Metazoa. (B) These topologies showed EGT in addition to the LGT from bacteria/archaea into Metazoa. See text for further details.

\section{TOPOLOGIES WITH TWO LGT EVENTS}

In addition to the presence/absence genes, we also found seven genes with topologies indicative of two interdomain LGT events: one into Metazoa and another into a different eukaryotic clade. Among the remaining 1517 single-gene trees after finding presence/absence genes, 15 gene trees showed topologies consistent with two interdomain LGT events involving Metazoa. We further used RAxML (Stamatakis 2014) (PROTGAMMALG model with default parameters, 100 bootstrap replicates) to bootstrap these trees and assess the support for the topological relationships. We found two topological trends: a metazoan clade and one other eukaryotic clade each nested within bacteria (Fig. 2A), and a metazoan clade and another clade of two photosynthetic eukaryotic groups, one nested within the other (Fig. 2B). We required the Metazoa and other eukaryotic clade to be separated by a bootstrap value of 70 or greater to increase confidence in resulting data. These criteria resulted in detection of seven genes, each with two topologically well-supported LGT events.

\section{DETERMINING THE TIMING OF LGT EVENTS}

To determine the approximate time of transfer (i.e. placement on our phylogeny), we assumed that each gene was transferred into Metazoa once and into the last common ancestors of the taxa containing the gene. Any nested "missing" taxa were assumed to either represent a loss, which is consistent with evidence for rampant gene loss among eukaryotes (Wolf and Koonin 2013; Katz 2015) or to be due to failure to capture in incomplete sampline (i.e. transcriptome data). For example, if the gene was found in 


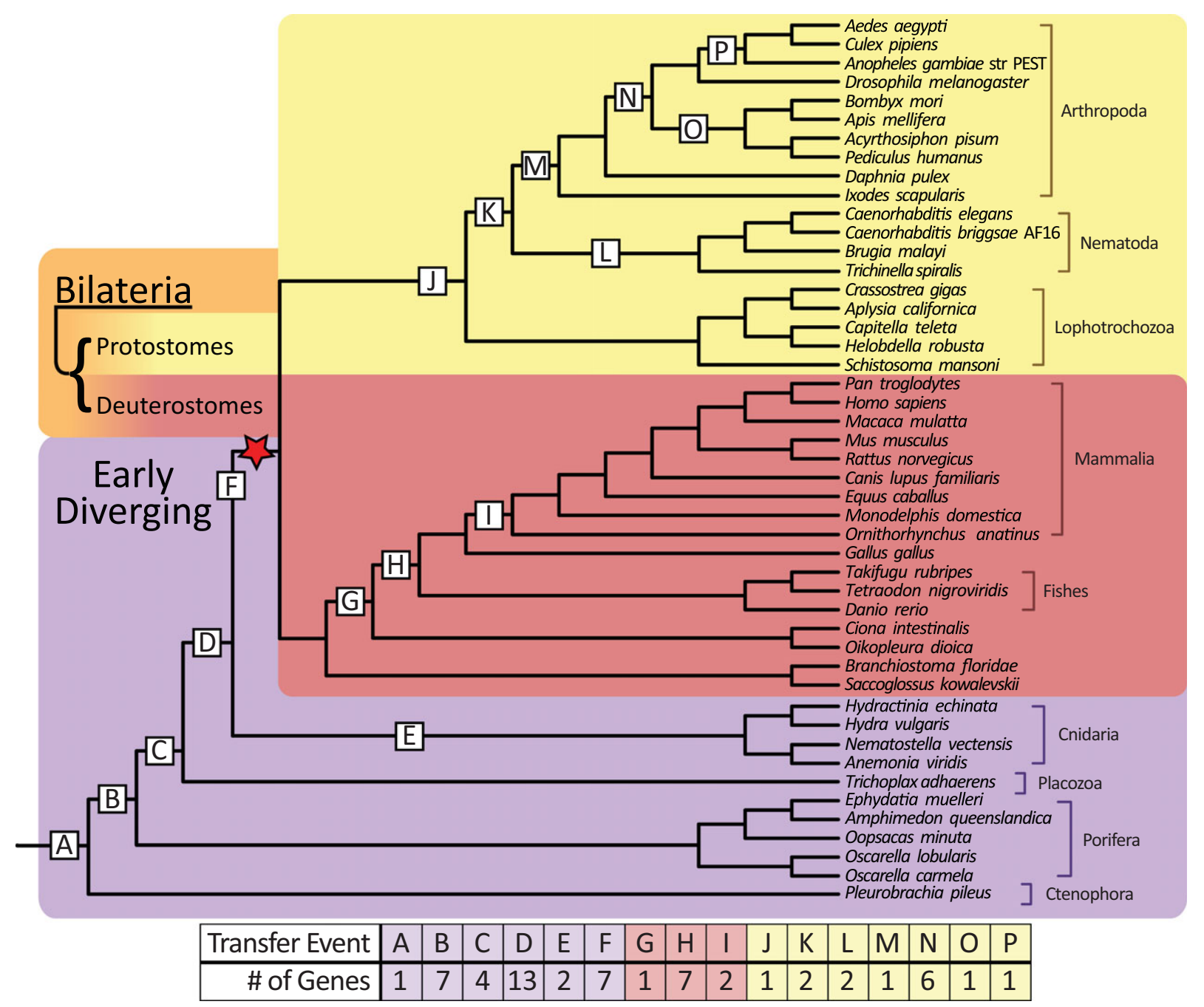

Figure 3. Estimate of LGTs across the metazoan tree of life. We charted LGTs onto a synthetic tree of metazoan evolution to show the relative time of transfer for each gene analyzed in this study. The synthetic tree includes only the Metazoa whose genomes contained one of the $\mathbf{5 8}$ laterally transferred genes found in this study (see Table S1 for a complete list of metazoan taxa in the pipeline). The letters in the square boxes are categories that bin the LGT events found in this study by the relative time of transfer. The table at the bottom provides a numerical summary of how many genes were found per event, and the letters correspond to Table 1 and Table S2, which contains more detailed information on the genes at each event. The star denotes when a sequestered germline developed. See text for detailed information on how this synthetic tree was created. Branch lengths are not proportional to time.

Cnidaria and bilateria, we inferred the LGT event occurred before the divergence of cnidarians and bilaterians (Fig. 3; event D). An alternative here would be to infer one interdomain LGT followed by LGT within Metazoa, which is possible but less likely. As another example, a gene found only in Cnidaria was assumed to have been transferred after the divergence of this lineages (Fig. 3 ; event $\mathrm{E}$ ). We recognize that our approach will misrepresent the timing of LGT events that occurred early and were then lost or not yet sampled in nontriploblastic lineages.

For the purpose of visualizing the approximate transfer times for the genes we found, we created the synthetic tree shown in Figure 3. We generated this phylogeny of the metazoan taxa included in our pipeline by concatenating vertically transferred genes and building a phylogeny in RAxML. Then, the tree was adjusted to reflect the arrangement of major metazoan phyla found in Ryan et al. (2013), creating a synthetic tree of relationships among taxa in our pipeline. To illustrate the approximate timing of events, branches with an LGT event was assigned a letter (Fig. 3) corresponding to Tables 1 and $\mathrm{S} 2$.

\section{ASSESSING GENE FUNCTION}

A representative sequence from each of the 58 presence/absence genes was passed through the KEGG BLAST tool (Kanehisa and Goto 2000; where KEGG is Kyoto encyclopedia of genes and genomes) to determine gene names and functions based upon the KEGG BRITE Hierarchy Ontology. We used a human or 
other "model" organism as the representative sequence for a gene when available. If multiple functional categories were available, we choose the function that appeared most applicable to both bacteria/archaea and metazoan, though certainly functions have changed over time.

\section{Results}

Consistent with the hypothesis that a sequestered germline creates an obstacle to LGT in Metazoa (e.g., Andersson 2005; Keeling and Palmer 2008), our analyses reveal a greater number of gene transfers before the development of a sequestered germline in bilaterian animals. A total of 58 genes satisfied our "presence/absence" criteria for LGT as they are only found in bacteria/archaea and Metazoa (Table 1, Fig. 1). Of those 58 interdomain LGTs, we found 34 genes transferred prior to the evolution of the sequestered germline (Table 1, Fig. 3; events A-F) as these genes were present in at least one of the 25 early-diverging lineages included in our analyses (Table S1). In contrast, we only detect 24 LGT events restricted to Bilateria (Table 1, Fig. 3; events G-P), where we had 36 species sampled (Table S1). If the detection of LGT events is dependent only on the number of species sampled, then the distributions-34 LGTs into 25 species and 24 LGTs into 36 species - are significantly different based on goodness-of-fit $G$-test $(P$-value $=0.0069)$, with an overabundance of events into early-diverging lineages.

Only one gene (OG5_139285, RENBP) appears to have been transferred from prokaryotes into the last common ancestor of Metazoa (Tables 1 and S2, Fig. 3; event A). Of the remaining 33 genes, our analyses reveal that seven genes were transferred before the divergence of sponges (Table 1, Fig. 3; event B), four before the divergence of Placozoa (Table 1, Fig. 3; event C), 13 before the divergence of cnidarians (Table 1, Fig. 3; event D), and seven before the divergence of protostomes and deuterostomes (Table 1, Fig. 3; event F); though interpretation must be made with caution given debates on relationships among these early lineages (Edgecombe et al. 2011; Ryan et al. 2013; Shu et al. 2014; Just et al. 2014). Finally, two laterally transferred genes were found in Cnidaria alone (Fig. 3; event E).

Within Bilateria, we found a total of 24 genes laterally transferred into either protostomes or deuterostomes and thus after acquisition of a sequestered germline at the base of Bilateria. Ten of these genes were found in only deuterostomes, whereas the other 14 were found only in protostomes (Table 1). Further, seven of the 10 were present only in vertebrates (fishes, mammals, and birds; Fig. 3; event $\mathrm{H}$ ) and two genes were present exclusively in mammals (Fig. 3; event I). Of the 14 genes laterally transferred into protostomes, only one gene was transferred before the split of Lophotrocozoa and Ecdysozoa (Fig. 3; event J); the remaining 13 were present exclusively in Ecdysozoa. Nine of those 13 were transferred into arthropods (Fig. 3; events M-P). Two genes were present in just nematodes (Fig. 3; event L) and two genes appear to have been transferred before the split of nematodes and arthropods (Fig. 3; event K).

Out of the seven gene topologies supporting two LGT events (i.e., genes present in bacteria/archaea, Metazoa, and one other eukaryotic group), all seven were transferred before the development of a sequestered germline. Two trends within the topologies emerged upon examination: the nonmetazoan monophyletic branch contained eukaryotes of a single major clade (often parasitic, e.g., Entamoeba and Trichomonas vaginalis; Fig. 2A) or consisted of two plastid-containing eukaryotes (one nested within the other, such as haptophytes and photosynthetic dinoflagellates; Fig. 2B). The trees showing transfers into plastid-containing eukaryotes suggest that these genes may be the result of EGT into the photosynthetic lineages.

\section{LATERALLY TRANSFERRED GENES SERVE PRIMARILY METABOLIC FUNCTIONS}

We used KEGG classification to assign putative gene functions for 34 of the 58 genes, with the remaining gene functions unknown/hypothetical. Of the 34 genes with known functions, the majority (16) are metabolic (Tables 2 and S2). Transporters made up the next most numerous category with 13 genes, eight of which belonged to the solute carrier family (Table 2). The remaining genes represent diverse functions: DNA repair (2), genetic information processing (1), spliceosome-associated (1), and signal transduction (1) (Table 2). The large number of unknown/hypothetic genes may represent genes with lineage-specific functions.

\section{Discussion}

\section{ESTIMATED RATES OF LGT ARE AFFECTED BY GERMLINE SEQUESTRATION AS WELL AS BY GENE LOSS, GENOME SIZE, AND DATA QUALITY}

Our data reveal that the evolution of a sequestered germline is associated with a decrease in the rate of interdomain LGT (Fig. 3, Table 1). We find a greater proportion of interdomain LGTs (34/58) into lineages that predate the evolution of a sequestered germline even though we sampled fewer of these lineages than Bilateria ( 25 vs. 36 species, respectively; Table 1; Fig. 1). Additional biases in our data include a greater number and higher quality data (i.e., more complete genomes) from Bilateria, and both a longer time span for gene loss and tendency for smaller genomes in earlydiverging lineages. Despite these, we found that the number of genes transferred into Metazoa before the evolution of a sequestered germline is significantly different $(P$-value $<0.01)$ 
Table 1. Number of genes transferred before and after germline sequestration and notes.

\begin{tabular}{llll}
\hline Recipient & Number of genes & Details \\
\hline Early diverging & 34 & $\begin{array}{c}\text { Before the divergence of: ctenophores (one), sponges (seven), } \\
\text { placozoa (four), cnidarians (13; and two after divergence), } \\
\text { protostomes, and deuterostomes (seven) }\end{array}$ \\
& $\begin{array}{l}\text { Deuterostomes } \\
\text { Protostomes }\end{array}$ & 10 & $\begin{array}{l}\text { Arthropods (nine), nematodes (two), other (three) } \\
\text { Total }\end{array}$ \\
\hline
\end{tabular}

For detailed information on approximately when the transfers occurred, see Figure $\mathbf{3}$ and Table S2.

Table 2. Protein function categories.

\begin{tabular}{lll}
\hline Function & $\begin{array}{c}\text { Number } \\
\text { of genes }\end{array}$ & Notes \\
\hline Metabolism & 16 & $\begin{array}{c}\text { Related to carbohydrates, lipids, nucleotides, amino acids, cofactors and vitamins, and } \\
\text { others } \\
\text { Eight are members of the Solute carrier family }\end{array}$ \\
$\begin{array}{l}\text { Transporters } \\
\text { DNA repair }\end{array}$ & 13 & \\
$\begin{array}{l}\text { Genetic information } \\
\text { processing }\end{array}$ & 1 & \\
$\begin{array}{l}\text { Signal transduction } \\
\text { Spliceosome-associated }\end{array}$ & 1 & \\
\hline Unknown & 24 & \\
\hline
\end{tabular}

For detailed information on the individual genes, see Table S2.

than the number of genes transferred into metazoans with a sequestered germline.

Previous claims that LGT is rare in Metazoa have generally failed to consider the fact that not all metazoans have a sequestered germline. Early-diverging metazoan lineages generally lack a sequestered germline and there is a greater chance for their gamete-producing cells to come in contact with foreign DNA. For example, cnidarian gonads are often located in the gastrodermis, the interior lining of the cnidarian "gut" or other structures close to the gut (Wedi and Dunn 1983; Eckelbarger et al. 1998; Brusca and Brusca 2003; Galliot et al. 2006; NishimiyaFujisawa and Kobayashi 2012). In ctenophores, the gonads form along different "canals" depending upon the species, all of which connect to the "gut" at some point (Komai 1922; Harbison and Miller 1986; Brusca and Brusca 2003). Although poriferans have no designated gonads, two cell types are typically responsible for transforming into gametes: (1) the choanocytes that line the internal water-filtering systems and are responsible for catching and ingesting nutrients and (2) archeocytes, a totipotent amoeboid cell that moves throughout the body of the sponge (Tsurumi and Reiswig 1997; Simpson 1980, 1984; Gaino et al. 1984, 1986; Brusca and Brusca 2003; Funayama 2010). As such, the gameto- genic cells in these early-diverging lineages either exist in close quarters to digestion, an area likely to come in contact with foreign DNA, or are responsible for ingestion themselves, all of which could increase their rate of LGT in comparison to bilaterian lineages.

An alternative explanation for the larger proportion of genes transferred into early-diverging lineages is that early-diverging Metazoa have been around longer and thus have had more time for LGT to occur. Although the exact dating and divergence of Metazoa is debated (Edgecombe et al. 2011; Shu et al. 2014), Metazoa likely first emerged around 800 million years ago (mya), whereas the divergence of Bilateria occurred approximately 550 mya (Erwin et al. 2011), leaving 250 mya for early-diverging metazoans to acquire more genes from bacteria (as well as an extra $\sim 250$ million years to lose genes; see next paragraph). However, we sampled more bilaterian than early-diverging lineages and the species richness of bilaterian lineages (particularly Arthropoda) dwarf that of early-diverging metazoans (Zhang 2013). Both these factors should bias toward more detection of LGT among Bilateria, even given our requirement that an LGT event be found in at least two descendant lineages. Instead, our analyses show that a larger number of genes were transferred into Metazoa during the 
smaller time span before the evolution of a sequestered germline, whereas we found fewer genes transferred into Bilateria since the development of a germline. Our data reveal that at least 32 genes were transferred from prokaryotes in the relatively short $\sim 250$ million years between the divergence of Metazoa and the development of a sequestered germline $(\mathrm{A}+\mathrm{B}+\mathrm{C}+\mathrm{D}+\mathrm{F}$, all transfers that were present in Early Diverging and bilaterian taxa), whereas only 24 genes were transferred into Bilateria in the $\sim 550$ million years since.

An additional caveat that may lead to an underestimate of early LGT events is the relatively small genomes in the sampled early-diverging metazoan lineages. For example, 11 of the deuterostome genomes included in this study have one billion or more base pairs compared to just two of the protostomes and two of the early-diverging lineages (Gregory 2016). Given that gene loss is so common throughout evolution (Keeling and Palmer 2008; Wolf and Koonin 2013; Katz 2015), it is possible that many LGTs are lost during genomic streamlining in metazoans with small genomes, like those in the sampled early-diverging species. Conversely, the larger genomes found throughout Bilateria might create the opposite problem, particularly in the deuterostomes: larger genomes may undergo less genomic streamlining (Gregory 2001; Lynch and Conery 2000; Wolf and Koonin 2013), resulting in more LGT-tolerant genomes (Table $\mathrm{S} 1$ ) and possibly an overestimate of LGTs. For example, the apparent pulse of transfer events in vertebrates (Table 1, Fig. 3; event $\mathrm{H}$ ) could be explained as either independent LGTs into deuterostomes, or the retention of genes transferred sometime before the divergence of deuterostomes and protostomes but then lost in lineages with smaller genomes.

Finally, the quality of the data also affects the patterns found in this study even though we used strict criteria for interpreting interdomain LGTs. Many of the early-diverging taxa do not yet have whole genome sequences available and, as a result, we used ESTs or RNAseq data. As such, genes transferred before the development of a sequestered germline may not have been present in these incomplete datasets. For example, the 13 genes in event D (Fig. 3) may have been transferred sometime before the divergence of cnidarians, but may not have been detected in our analyses due to poor sequencing data from early-diverging lineages. Overall, the quality and quantity of bilaterian genomic data are better than for early diverging lineages, which makes finding laterally transferred genes in early diverging lineages more challenging.

When combined with our strict criteria (i.e., "presence/absence" genes found only in prokaryotes and at least two metazoan), the differences in the quality of the underlying data likely lowered the number of inferred LGTs found among individual early-diverging phyla (e.g., Fig. 3; event E). For example, our criteria require that the gene be present in at least two Metazoa, but because sequencing data are only available for one species in phylum Placozoa (Trichoplax adhaerens), we cannot detect any ancient LGT that occurred in this lineage alone. In sum, the biased distribution of nonwhole genome data among early-diverging taxa likely leads to an underestimate of the role of a sequestered germline in blocking LGTs as early LGT events are more likely to go undetected.

\section{LIFE HISTORY COULD RESULT IN "WEAK-LINKS" AND HIGHER CHANCES OF LGT AMONG DIFFERENT LINEAGES}

Lateral gene transfers might also be enhanced in lineages that have life-history stages with greater exposure to potential donor lineages (i.e., the "weak-link model" [Huang 2013]). Weak links associated with specific lifestyles could include harboring endosymbionts or parasites (e.g., Wolbachia in arthropods and nematodes), external fertilization, and the retention of totipotent cells throughout adulthood (often associated with regeneration and asexual reproduction; Huang 2013). Many early-diverging Metazoa exhibit these traits (Tsurumi and Reiswig 1997; Extavour et al. 2005; Galliot et al. 2006; Funayama 2010; Juliano et al. 2010; David 2012; Nishimiya-Fujisawa and Kobayashi 2012), but some Bilateria do as well, including Planaria (Newmark et al. 2008; Agata and Inoue 2012), some species of newt (Okamoto et al. 2007), echinoderms (Shibata et al. 2010), and others (Newmark et al. 2008; Giani et al. 2011; Collins et al. 2013). In particular, bdelloid rotifers serve as an exceptional example of a "weak-link" lifestyle leading to rampant LGT in a protostome; bdelloid rotifers survive desiccation and genome decay periodically, which likely makes incorporating laterally transferred genes into the genome much easier (Flot et al. 2013). Indeed, recent studies examining several species of bdelloid rotifers have found that large portions of their genomes are from foreign sources (Boschetti et al. 2012; Flot et al. 2013; Eyres et al. 2015). Although we did not include any bdelloid rotifers in our study, we do agree that bdelloid rotifers serve as a clear example of "weak-link" taxa with increased potential for LGT. We did sample two bilaterian species (Schistosoma mansoni and Capitella teleta) exhibiting weak-link traits, and we find no evidence of increased LGTs into these lineages (Fig. 3), possibly because we did not include LGTs into a single species. However, we predict that additional examples will be found with the emergence of data from more diverse lineages.

\section{COMPARISONS OF OUR INSIGHTS TO OTHER STUDIES}

We also used our pipeline to examine laterally transferred genes reported in other studies (Boschetti et al. 2012; Crisp et al. 2015). Upon examining 394 genes individually reported to be found in Homo sapiens, Drosophila melanogaster, and Caenorhabditis elegans, we found that nearly half (177) of these genes mapped to the same homologous group (i.e., they represent paralogs in 
a single 'OG' (Chen et al, 2006)) leaving 217 putative laterally transferred genes. Of these, 97 gene trees were found in our pipeline. Using custom Python scripts, we asked to what degree the remaining 97 gene trees satisfied our criteria for LGT between bacteria/archaea and metazoan. Only three genes passed our conservative criteria for interdomain LGT and are included and reported in this study. Two are part of the 58 presence/absence genes (Fig. 3 and Table S2; event L), while one is in the reported topologies with more than one LGT (a gene named NQO1, transferred before deuterostomes and protostomes diverged, OG5_138113). The remaining genes did not match our criteria as they are either not found in more than one metazoan "recipient" lineage or are found in gene trees with other eukaryotes; again, we choose to focus only on interdomain LGTs given the systematic errors that can lead to incorrect topologies in single gene trees capturing hundreds of millions of years of evolution.

The small overlap between our final dataset and the datasets of Crisp et al. (2015) and Boschetti et al. (2012) is expected. It is common for studies that use different methodologies and criteria to come up with different final datasets (Ragan 2001; Lawrence and Ochman 2002; Lawrence and Hendrickson 2003; Dagan 2011). Also, because these studies considered intradomain LGT, many of the genes found in Crisp et al. (2015) and Boschetti et al. (2012) are also present in nonmetazoan eukaryotes and would have been removed under our criteria for interdomain LGTs. Furthermore, both Boschetti et al. (2012) and Crisp et al. (2015) used surrogate methods with unique parameters to search for LGTs in C. elegans and reported different numbers of found genes between themselves. Ultimately, all three studies (Boschetti et al. 2012; Crisp et al. 2015; and our own) used different approaches and parameters to address distinct questions, making it difficult to accurately compare across the three.

\section{THE MAJORITY OF PROTEINS INVOLVED IN LGT EVENTS HAVE METABOLIC OR TRANSPORT ROLES}

Although only 34 of the 58 presence/absence genes had known functions, the majority of these (16) are involved in metabolism, with the remaining 18 genes scattered into a few other functional categories (Table 2). This is consistent with the types of genes transferred among bacteria (Pál et al. 2005). Although LGT has been considered a source of adaptive traits (Schönknecht et al. 2014), the majority of LGTs tend to serve metabolic functions, perhaps because these genes are able to integrate into comparatively simple metabolic gene networks as opposed to more complicated ones (Jain et al. 1999). Transporters were the next most numerous (13 genes) functional category found, likely because membrane transport genes are quite common. Membrane proteins themselves make up $27 \%$ of all human protein-coding genes, of which the solute carrier family is the second largest family with at least 50 defined families (Fredriksson et al. 2008; Almen et al.
2009). The eight solute carrier family genes found here (Tables 1 and S2) were from a variety of gene families.

\section{LIMITATIONS AND FUTURE WORK}

The methodology we used in this study enabled us to find instances of interdomain LGT in lineages across the metazoan tree of life. However, we acknowledge that there are numerous limitations to our analyses. For example, some taxa have only transcriptome data or limited amounts of whole genome data available, and these biases are likely greater among early-diverging lineages. Moreover, our inferences are dependent on our taxon sampling. We assembled a sampling of Metazoa that balanced phylogenetic breadth and evenness; there are many more triploblast lineages that we could have sampled and inclusion of these would surely increase the number of recent LGT events inferred. However, earlydiverging lineages lack of similar levels of sampling and so creating additional unevenness would introduce further bias. Future studies with additional taxa will surely change the details of inferences on LGTs as increasing taxon sampling from lineages across the metazoan tree of life will increase the power of the analyses.

\section{DATA ARCHIVING}

The doi for our data is http://dx.doi.org/10.5061/dryad.6d3v0

\section{LITERATURE CITED}

Agata, K., and T. Inoue. 2012. Survey of the differences between regenerative and non-regenerative animals. Dev. Growth Diff. 54:143-152. doi:10.1111/j.1440-169X.2011.01323.x.

Alie, A., L. Leclere, M. Jager, C. Dayraud, P. R. Chang, H. Le Guyader, E. Queinnec, and M. Manuel. 2011. Somatic stem cells express Piwi and Vasa genes in an adult ctenophore: ancient association of "germline genes" with stemness. Dev. Biol. 350:183-197. doi:10. 1016/j.ydbio.2010.10.019.

Almen, M. S., K. J. V. Nordstrom, R. Fredriksson, and H. B. Schioth. 2009. Mapping the human membrane proteome: a majority of the human membrane proteins can be classified according to function and evolutionary origin. BMC Biol. 7:14. doi:10.1186/1741-7007-7-50.

Altschul, S. F., W. Gish, W. Miller, E. W. Myers, and D. J. Lipman. 1990. Basic local alignment search tool. J. Mol. Biol. 215:403-410.

Andersson, J. O. 2005. Lateral gene transfer in eukaryotes. Cell. Mol. Life Sci. 62:1182-1197. doi:10.1007/s00018-005-4539-z.

Andersson, J. O., W. F. Doolittle, and C. L. Nesbø. 2001. Are there bugs in our genome? Science 292:1848-1850

Boschetti, C., A. Carr, A. Crisp, I. Eyres, Y. Wang-Koh, E. Lubzens, T. G. Barraclough, G. Micklem, and A. Tunnacliffe. 2012. Biochemical diversification through foreign gene expression in bdelloid rotifers. PLoS Genet. 8:e1003035. doi:10.1371/journal.pgen.1003035.

Boto, L. 2010. Horizontal gene transfer in evolution: facts and challenges. Proc. R. Soc. B Biol. Sci. 277:819-827. doi:10.1098/rspb.2009.1679.

. 2014. Horizontal gene transfer in the acquisition of novel traits by metazoans. Proc. R. Soc. B Biol. Sci. 281:20132450. doi:10.1098/rspb.2013.2450

Brusca, R. C., and G. J. Brusca. 2003. Invertebrates. Sinauer, Sunderland. Chapman, J. A., E. F. Kirkness, O. Simakov, S. E. Hampson, T. Mitros, T. Weinmaier, T. Rattei, P. G. Balasubramanian, J. Borman, D. Busam, 
et al. 2010. The dynamic genome of Hydra. Nature 464:592-596. doi: 10.1038 /nature 08830 .

Chen, F., A. J. Mackey, C. J. Stoeckert, and D. S. Roos. 2006. OrthoMCL-DB: querying a comprehensive multi-species collection of ortholog groups. Nucleic Acids Res. 34(Suppl. 1):D363-D368.

Cobbs, C., J. Heath, J. O. Stireman, and P. Abbot. 2013. Carotenoids in unexpected places: gall midges, lateral gene transfer, and carotenoid biosynthesis in animals. Mol. Phylogenet. Evol. 68:221-228.

Collins, J. J., B. Wang, B. G. Lambrus, M. E. Tharp, H. Iyer, and P. A. Newmark. 2013. Adult somatic stem cells in the human parasite Schistosoma mansoni. Nature 494:476-479. doi:10.1038/nature11924.

Crisp, A., C. Boschetti, M. Perry, A. Tunnacliffe, and G. Micklem. 2015. Expression of multiple horizontally acquired genes is a hallmark of both vertebrate and invertebrate genomes. Genome Biol. 16:50. doi:10.1186/s13059-015-0607-3.

Dagan, T. 2011. Phylogenomic networks. Trends Microbiol. 19:483-491.

Dagan, T., and W. Martin. 2009. Getting a better picture of microbial evolution en route to a network of genomes. Phil. Trans. Biol. Sci. 364:218702196.

Danchin, E. G. J., M.-N. Rosso, P. Vieira, J. de Almeida-Engler, P. M. Coutinho, B. Henrissat, and P. Abad. 2010. Multiple lateral gene transfers and duplications have promoted plant parasitism ability in nematodes. Proc. Natl. Acad. Sci. 107:17651-17656. doi:10. 1073/pnas. 1008486107.

David, C. N. 2012. Interstitial stem cells in Hydra: multipotency and decisionmaking. Int. J. Dev. Biol. 56:489-497. doi:10.1387/ijdb.113476cd.

Davison, J. 1999. Genetic exchange between bacteria in the environment. Plasmid 42:73-91. doi:10.1006/plas.1999.1421.

Doolittle, W. F. 1999. Phylogenetic classification and the universal tree. Science 284:2124-2128. doi:10.1126/science.284.5423.2124.

Dunning Hotopp, J. C. 2011. Horizontal gene transfer between bacteria and animals. Trends Genet. 27:157-163. doi:10.1016/j.tig.2011.01.005.

Eckelbarger, K. J., P. A. Tyler, and R. W. Langton. 1998. Gonadal morphology and gametogenesis in the sea pen Pennatula aculeata (Anthozoa: Pennatulacea) from the Gulf of Maine. Mar. Biol. 132:677690.

Edgecombe, G. D., G. Giribet, C. W. Dunn, A. Hejnol, R. M. Kristensen, R. C. Neves, G. W. Rouse, K. Worsaae, and M. V. Sorensen. 2011. Higherlevel metazoan relationships: recent progress and remaining questions. Org. Divers. Evol. 11:151-172. doi:10.1007/s13127-011-0044-4.

Erwin, D. H., M. Laflamme, S. M. Tweedt, E. A. Sperling, D. Pisani, and K. J. Peterson. 2011. The Cambrian Conundrum: early divergence and later ecological success in the early history of animals. Science 334:10911097. doi:10.1126/science. 1206375.

Ewen-Campen, B., E. E. Schwager, and C. G. M. Extavour. 2010. The molecular machinery of germ line specification. Mol. Reprod. Dev. 77:3-18. doi:10.1002/mrd.21091.

Extavour, C. G., and M. Akam. 2003. Mechanisms of germ cell specification across the metazoans: epigenesis and preformation. Development 130:5869-5884. doi:10.1242/dev.00804.

Extavour, C. G., K. Pang, D. Q. Matus, and M. Q. Martindale. 2005. vasa and nanos expression patterns in a sea anemone and the evolution of bilaterian germ cell specification mechanisms. Evol. Dev. 7:201-215. doi:10.1111/j.1525-142X.2005.05023.x.

Extavour, C. G. M. 2007. Evolution of the bilaterian germ line: lineage origin and modulation of specification mechanisms. Integrative and Comparative Biology no. 47 (5):770-785. doi:10.1093/icb/icm027.

Eyres, I., C. Boschetti, A. Crisp, T. P. Smith, D. Fontaneto, A. Tunnacliffe, and T. G. Barraclough. 2015. Horizontal gene transfer in bdelloid rotifers is ancient, ongoing and more frequent in species from desiccating habitats. BMC Biol. 13:1.
Flot, J.-F., B. Hespeels, X. Li, B. Noel, I. Arkhipova, E. G. J. Danchin, A. Hejnol, B. Henrissat, R. Koszul, J.-M. Aury, et al. 2013. Genomic evidence for ameiotic evolution in the bdelloid rotifer Adineta vaga. Nature 500:453-457.

Fredriksson, R., K. J. V. Nordstrom, O. Stephansson, M. G. A. Hagglund, and H. B. Schioth. 2008. The solute carrier (SLC) complement of the human genome: phylogenetic classification reveals four major families. FEBS Lett. 582:3811-3816. doi:10.1016/j.febslet.2008.10.016.

Funayama, N. 2010. The stem cell system in demosponges: insights into the origin of somatic stem cells. Dev. Growth Diff. 52:1-14. doi:10.1111/j.1440-169X.2009.01162.x.

Gaino, E., B. Burlando, L. Zunino, M. Pansini, and P. Buffa. 1984. Origin of male gametes from choanocytes in Spongia officinalis (Porifera, Demospongiae). Int. J. Invertebr. Repr. Dev. 7:83-93.

Gaino, E., B. Burlando, and P. Buffa. 1986. Contribution to the study of egg development and derivation in Oscarella lobularis (Porifera, Demospongiae). Int. J. Invertebr. Repr. Dev. 9:59-69.

Galliot, B., M. Mijkovic-Licina, R. de Rosa, and S. Chera. 2006. Hydra, a niche for cell and developmental plasticity. Sem. Cell Dev. Biol. 17:492-502. doi:10.1016/j.semcdb.2006.05.005.

Giani, V. C., E. Yamaguchi, M. J. Boyle, and E. C. Seaver. 2011. Somatic and germline expression of piwi during development and regeneration in the marine polychaete annelid Capitella teleta. Evodevo 2:18. doi: 10.1186/2041-9139-2-10.

Gladyshev, E. A., M. Meselson, and I. R. Arkhipova. 2008. Massive horizontal gene transfer in bdelloid rotifers. Science 320:1210-1213. doi:10.1126/science.1156407.

Gogarten, J. P., W. F. Doolittle, and J. G. Lawrence. 2002. Prokaryotic evolution in light of gene transfer. Mol. Biol. Evol. 19:2226-2238.

Grant, J. R., and L. A. Katz. 2014. Building a phylogenomic pipeline for the eukaryotic tree of life - addressing deep phylogenies with genome-scale data. PLoS Curr. 6. doi:10.1371/currents.tol. c24b6054aebf3602748ac042ccc8f2e9.

Gregory, T. R. 2001. Coincidence, coevolution, or causation? DNA content, cell size, and the C-value enigma. Biol. Rev. 76:65-101. doi:10. 1111/j.1469-185X.2000.tb00059.x.

. 2016. Animal genome size database. Available at http://www.geno mesize.com.

Haegeman, A., J. T. Jones, and E. G. J. Danchin. 2011. Horizontal gene transfer in nematodes: a catalyst for plant parasitism? Mol. Plant Microbe Interact. 24:879-887. doi:10.1094/MPMI-03-11-0055.

Harbison, G. R., and R. L. Miller. 1986. Not all ctenophores are hermaphrodites. Studies on the systematics, distribution, sexuality and development of two species of Ocyropsis. Mar. Biol. 90:413-424.

Huang, J. 2013. Horizontal gene transfer in eukaryotes: the weak-link model. BioEssays 35:868-875. doi:10.1002/bies.201300007.

Jackson, D. J., L. Macis, J. Reitner, and G. Wörheide. 2011. A horizontal gene transfer supported the evolution of an early metazoan biomineralization strategy. BMC Evol. Biol. 11:238. doi:10.1186/1471-2148-11-238.

Jain, R., M. C. Rivera, and J. A. Lake. 1999. Horizontal gene transfer among genomes: the complexity hypothesis. Proc. Natl. Acad. Sci. USA 96:3801-3806.

Jain, R., M. C. Rivera, J. E. Moore, and J. A. Lake. 2003. Horizontal gene transfer accelerates genome innovation and evolution. Mol. Biol. Evol. 20:1598-1602. doi:10.1093/molbev/msg 154.

Juliano, C. E., S. Z. Swartz, and G. M. Wessel. 2010. A conserved germline multipotency program. Development 137:4113-4126. doi:10.1242/dev.047969.

Just, J., R. M. Kristensen, and J. Olesen. 2014. Dendrogramma, new genus, with two new non-bilaterian species from the marine bathyal of southeastern Australia (animalia, metazoa incertae sedis)—with similarities 
to some medusoids from the precambrian ediacara. Plos One 9: e102976. doi: 10.1371/journal.pone.0102976.

Kanehisa, M., and S. Goto. 2000. KEGG: Kyoto encyclopedia of genes and genomes. Nucleic Acids Res. 28:27-30.

Katz, L. A. 2002. Lateral gene transfers and the evolution of eukaryotes: theories and data. Int. J. Syst. Evol. Microbiol. 52:1893-1900. doi:10.1099/ijs.0.02113-0.

2012. Origin and diversification of eukaryotes. Ann. Rev. Microbiol. 66:411-427.

- 2015. Recent events dominate interdomain lateral gene transfers between prokaryotes and eukaryotes and, with the exception of endosymbiotic gene transfers, few ancient transfer events persist. Phil. Trans. R. Soc. B 370:20140324. doi:10.1098/rstb.2014.0324.

Keeling, P. J., and J. D. Palmer. 2008. Horizontal gene transfer in eukaryotic evolution. Nat. Rev. Genet. 9:605-618. doi:10.1038/nrg2386.

Klasson, L., Z. Kambris, P. E. Cook, T. Walker, and S. P. Sinkins. 2009. Horizontal gene transfer between Wolbachia and the mosquito Aedes aegypti. BMC Genom. 10:33. doi:10.1186/1471-2164-10-33.

Komai, T. 1922. Studies on two aberrant ctenophores: Coeloplana and Gastrodes. Author, Kyoto.

Kondrashov, F. A., E. V. Koonin, I. G. Morgunov, T. V. Finogenova, and M. N. Kondrashova. 2006. Evolution of glyoxylate cycle enzymes in Metazoa: evidence of multiple horizontal transfer events and pseudogene formation. Biol. Direct 1:1-14. doi:10.1186/1745-6150-1-31.

Koning, A. P. de, F. S. L. Brinkman, S. J. M. Jones, and P. J. Keeling. 2000. Lateral gene transfer and metabolic adaptation in the human parasite Trichomonas vaginalis. Mol. Biol. Evol. 17:1769-1773.

Lawrence, J. G., and H. Hendrickson. 2003. Lateral gene transfer: when will adolescence end? Mol. Microbiol. 50:739-749.

Lawrence, J. G., and H. Ochman. 2002. Reconciling the many faces of lateral gene transfer. Trends Microbiol. 10:1-4.

Lehmann, R., and B. E. Richardson. 2010. Mechanisms guiding primordial germ cell migration: strategies from different organisms. Nat. Rev. Mol. Cell Biol. 11. 37-49. doi:10.1038/nrm2815

Lynch, M., and J. S. Conery. 2000. The evolutionary fate and consequences of duplicate genes. Science 290:1151-1155. doi:10. 1126/science.290.5494.1151.

Nakashima, K., L. Yamada, Y. Satou, J.-i. Azuma, and N. Satoh. 2004. The evolutionary origin of animal cellulose synthase. Dev. Genes Evol. 214:81-88. doi:10.1007/s00427-003-0379-8.

Nelson, K. E., R. A. Clayton, S. R. Gill, M. L. Gwinn, R. J. Dodson, D. H. Haft, E. K. Hickey, J. D. Peterson, W. C. Nelson, K. A. Ketchum, et al. 1999. Evidence for lateral gene transfer between Archaea and Bacteria from genome sequence of Thermotoga maritima. Nature 399:323-329. doi:10.1038/20601.

Newmark, P. A., Y. Wang, and T. Chong. 2008. Germ cell specification and regeneration in planarians. Cold Spring Harb. Symp. Quant. Biol. 73:573581. doi:10.1101/sqb.2008.73.022.

Nikoh, N., and A. Nakabachi. 2009. Aphids acquired symbiotic genes via lateral gene transfer. BMC Biol. 7. 12 doi:10.1186/1741-7007-7-12.

Nishimiya-Fujisawa, C., and S. Kobayashi. 2012. Germline stem cells and sex determination in Hydra. Int. J. Dev. Biol. 56:499-508. doi:10.1387/ijdb.123509cf.

Ochman, H., J. G. Lawrence, and E. A. Groisman. 2000. Lateral gene transfer and the nature of bacterial innovation. Nature 405:299-304. doi: $10.1038 / 35012500$.

Okamoto, M., H. Ohsawa, T. Hayashi, K. Owaribe, and P. A. Tsonis. 2007. Regeneration of retinotectal projections after optic tectum removal in adult newts. Mol. Vis. 13:2112-2118.

Paganini, J., A. Campan-Fournier, M. Da Rocha, P. Gouret, P. Pontarotti, E. Wajnberg, P. Abad, and E. G. J. Danchin. 2012. Contribution of lateral gene transfers to the genome composition and parasitic ability of rootknot nematodes. PLoS One 7:e50875.

Pál, C., B. Papp, and M. J. Lercher. 2005. Adaptive evolution of bacterial metabolic networks by horizontal gene transfer. Nat. Genet. 37:13721375. doi:10.1038/ng1686.

Penn, O., E. Privman, G. Landan, D. Graur, and T. Pupko. 2010. An alignment confidence score capturing robustness to guide tree uncertainty. Mol. Biol. Evol. 27:1759-1767. doi:10.1093/molbev/msq066.

Price, M. N., P. S. Dehal, and A. P. Arkin. 2009. FastTree: computing large minimum evolution trees with profiles instead of a distance matrix. Mol Biol. Evol. 26:1641-1650. doi:10.1093/molbev/msp077.

2010. FastTree 2: approximately maximum-likelihood trees for large alignments. Plos One 5:10. doi:10.1371/journal.pone.0009490.

Ragan, M. A. 2001. On surrogate methods for detecting lateral gene transfer. FEMS Microbiol. Lett. 201:187-191.

Ragan, M. A., T. J. Harlow, and R. G. Beiko. 2006. Do different surrogate methods detect lateral genetic transfer events of different relative ages? Trends Microbiol. 14:4-8.

Ryan, J. F., K. Pang, C. E. Schnitzler, A. D. Nguyen, R. T. Moreland, D. K. Simmons, B. J. Koch, W. R. Francis, P. Havlak, S. A. Smith, et al. 2013. The genome of the ctenophore Mnemiopsis leidyi and its Implications for cell type evolution. Science 342:1336-1344. doi: 10.1126/science.1242592.

Schönknecht, G., W.-H. Chen, C. M. Ternes, G. G. Barbier, R. P. Shrestha, M. Stanke, A. Bräutigam, B. J. Baker, J. F. Banfield, and R. M. Garavito. 2013. Gene transfer from bacteria and archaea facilitated evolution of an extremophilic eukaryote. Science 339:1207-1210.

Schönknecht, G., A. P. M. Weber, and M. J. Lercher. 2014. Horizontal gene acquisitions by eukaryotes as drivers of adaptive evolution. BioEssays 36:9-20. doi:10.1002/bies.201300095.

Shibata, T. F., T. Oji, K. Akasaka, and K. Agata. 2010. Staging of regeneration process of an arm of the feather star Oxycomanthus japonicus focusing on the oral-aboral boundary. Dev. Dyn. 239:2947-2961. doi:10.1002/dvdy.22429.

Shu, D. G., Y. Isozaki, X. L. Zhang, J. Han, and S. Maruyama. 2014. Birth and early evolution of metazoans. Gondwana Res. 25:884-895. doi:10.1016/j.gr.2013.09.001.

Simpson, T. L. 1980. Reproductive processes in sponges—a critical-evaluation of current data and views. Int. J. Invertebr. Repr. 2:251-269.

. 1984. The cell biology of sponges. Vol. i-xiv. Pp. 1-662. Springer, New York.

Sloan, D. B., and N. A. Moran. 2012. Endosymbiotic bacteria as a source of carotenoids in whiteflies. Biol. Lett. 8:986-989.

Soucy, S. M., J. Huang, and J. P. Gogarten. 2015. Horizontal gene transfer: building the web of life. Nat. Rev. Genet. 16:472-482.

Starcevic, A., S. Akthar, W. C. Dunlap, J. M. Shick, D. Hranueli, J. Cullum, and P. F. Long. 2008. Enzymes of the shikimic acid pathway encoded in the genome of a basal metazoan, Nematostella vectensis, have microbial origins. Proc. Natl. Acad. Sci. 105:2533-2537. doi:10.1073/pnas.0707388105.

Starz-Gaiano, M., and R. Lehmann. 2001. Moving towards the next generation. Mech. Dev. 105:5-18. doi:10.1016/S0925-4773(01)00392-6.

Syvanen, M. 2012. Evolutionary implications of horizontal gene transfer. Ann. Rev. Genet. 46:341-358. doi:10.1146/annurev-genet-110711155529.

Tsurumi, M., and H. M. Reiswig. 1997. Sexual versus asexual reproduction in an oviparous rope-form sponge, Aplysina cauliformis (Porifera; Verongida). Invertebr. Reprod. Dev. 32:1-9. doi:10.1080/ 07924259.1997.9672598.

Wedi, S. E., and D. F. Dunn. 1983. Gametogenesis and reproductive periodicity of the subtidal sea anemone Urticina lofotensis (Coelenterata: Actiniaria) in California. Biol. Bull. 165:458-472. 
Whitaker, J. W., G. A. McConkey, and D. R. Westhead. 2009. The transferome of metabolic genes explored: analysis of the horizontal transfer of enzyme encoding genes in unicellular eukaryotes. Genome Biol. 10:1-13. doi:10.1186/gb-2009-10-4-r36.

Wijayawardena, B. K., D. J. Minchella, and J. A. DeWoody. 2013. Hosts, parasites, and horizontal gene transfer. Trends Parasitol. 29:329-338.
Wolf, Y. I., and E. V. Koonin. 2013. Genome reduction as the dominant mode of evolution. Bioessays 35:829-837.

Zhang, Z. Q. 2013. Animal biodiversity: an update of classification and diversity in 2013. Zootaxa 3703:5-11.

Associate Editor: R. Redfield Handling Editor: J. Conner

\section{Supporting Information}

Additional Supporting Information may be found in the online version of this article at the publisher's website:

Figure S1. Visualization of our methodology.

Table S1. List of Metazoa, phyla, common names, data types, and genome sizes.

Table S2. Gene and protein name, functional five category, and approximate time of six transfer. 